\title{
Improving the Summation Law for Harmonic Current Emissions of Parallel Operated PV Inverters by Considering Equivalent Grid Impedance
}

\author{
Hasanali Moghadam, Florian Ackermann and Sönke Rogalla \\ Department Power Electronics and Grid Technologies \\ Fraunhofer Institute for Solar Energy Systems ISE \\ Heidenhofstr. 2, 79110 Freiburg (Germany) \\ Phone number: +49 (0) 761 4588-5947, e-mail: hasanali.moghadam@ise.fraunhofer.de
}

\begin{abstract}
For connection of a PV park to the electricity network, a pre-compliance study has to prove that the harmonic emissions at the point of common coupling (PCC) stays below the limits specified by the standards or local or national grid codes. An equation defined in IEC 61400-21 [1] is used to estimate the total harmonic current emission of the park at the PCC prior to installation. The equation uses the harmonic emission of a single inverter to predict the spectrum of the total current for a given number of identical inverters connected in parallel at the PCC.

This paper considers the interaction of parallel operated inverters and the grid impedance. It proposes an approach based on the so called "equivalent grid impedance", to improve the performance of summation law for evaluation of the current harmonics emissions at the PCC of PV parks. Improvement of this method would be helpful for power plant constructors.
\end{abstract}

\section{Key words}

Grid equivalent impedance, harmonic emissions, point of common coupling, photovoltaic inverter, summation law

\section{Introduction}

The increasing share of renewable energies in power systems implies the higher share of power electronicsbased generation units with their nonlinear characteristics. This raises the need to observe their power quality features more accurately, especially with respect to their harmonic emission. In this regard, all generation units have to comply with the predefined levels for the voltage and current harmonics stated in standards as it is discussed in [2].

Different criteria are proposed to evaluate the harmonic compatibility of the units. Most of the standards propose maximum permissible harmonic current levels as in IEC 61000-3-6 [3].

Several investigations during the previous decades have proposed empirical methods and probabilistic approach [4-6] to find a comprehensive method for calculating the aggregated current or voltage harmonic production of the generation units connected to the PCC.

The IEC 61400-21 [1] proposes a mathematical method to compute the overall harmonic current emissions of a PV park at the PCC before installation of the power plant. The computed values are then compared to the limits defined in standards (for example IEC 61000-3-6) and serves as a basis for issuing emission certificate for a power plant.

Investigations [7-9] have shown the shortcomings of this calculation method and reported higher forecasted emissions in comparison to the real measurements. However, this method still is the basis to predict the harmonic emission at the PCC).

In this paper, consideration of grid equivalent impedance introduced in [10] and [11] is proposed for measurements in order to investigate the harmonic behavior of inverters and eventually improving the results of the summation law. Lab measurements are performed to validate the proposed theory. This analysis can contribute to better performance of the existing standard and revision of the certification measurements for emission of PV parks.

\section{IEC Harmonic Summation Law and Prospect of its Application}

The most widely used method for summation of harmonics at PCC as recommended by IEC 61400-21 is introduced in Equation (1). This equation was originally introduced to estimate the global emission of parallel operated loads and later used for wind and PV production units. In this approach, the generation unit is assumed to be a current source and a mathematical solution obtained from an empirical approach, considering individual coefficient for each harmonic order, is proposed to calculate superposition of harmonics at the PCC.

$$
I_{h s}=\sqrt[\beta]{\sum_{i=1}^{N_{f}}\left(\frac{I_{h, i}}{n_{i}}\right)^{\beta}},
$$

Where $\mathrm{N}$ is the number of harmonic sources connected at the PCC, $I_{h, i}$ is the magnitude of the $h^{\text {th }}$ harmonic generated by the $i^{\text {th }}$ source, and $n_{i}$ is the transformation ratio assigned to the $i^{\text {th }}$ source, if there is a transformer 
between the source and the PCC measurement points. The $\beta$ values are defined as follows:

$$
\beta= \begin{cases}1.0 ; & \text { if } h<5 \\ 1.4 ; & \text { if } 5 \leq h \leq 10 \\ 2.0 ; & \text { if } h>10\end{cases}
$$

In order to use the summation law, the calculation of harmonics shall comply with the IEC 61000-4-7 standard wich considers the frequency range of up to $9 \mathrm{kHz}$. Discrete Fourier Transformation (DFT) is applied on the samples using 10 period windows (assuming $50 \mathrm{~Hz}$ ). The standard divides the frequency components into three categories: main order harmonics, inter-harmonics and higher order harmonics. A different grouping or subgruping procedure is then applied for each harmonic category and the process repeated for each DFT Window. The grouped or sub-grouped results are finally aggregated over a predefined number of DFT windows, typically 300 (one minute) or 3000 (10 minutes).

Equation 1 is finally applied to those aggregated and grouped or sub-grouped harmonic current emissions. Investigations such as [13] have reported a poor consistency between the measurements and the calculation results.

Studies [14] have discussed the possible backgrounds of this mismatch. Background distortion of the coupled electrical network and neglected effect of network impedance can have considerable influences on current harmonic emissions.

\section{Proposed Approach}

\section{A. Motivation}

The current and voltage harmonic spectra of an inverter depend not only on the inverter's behavior and its internal impedance but also on the impedance of the coupled network. Different network impedances can result in different harmonic spectrums [15]. A test bench with an inverter connected through a variable series impedance to a high bandwidth grid simulator with a THDU $<0.5 \%$ could help us to visualize the effect of the grid impedance on the current harmonic spectrum of the inverter. Single line diagram of this test bench is illustrated in Fig. 1.

The inverter is a $50 \mathrm{~kW}$, three phase device with neutral connection (four-wire). The series inductance $\left(\mathrm{L}_{\text {series }}\right)$ can be changed stepwise leading to five different values of the grid impedance. The effect of the variation of series Inductance on the current spectrum can be seen in Fig. 2 .

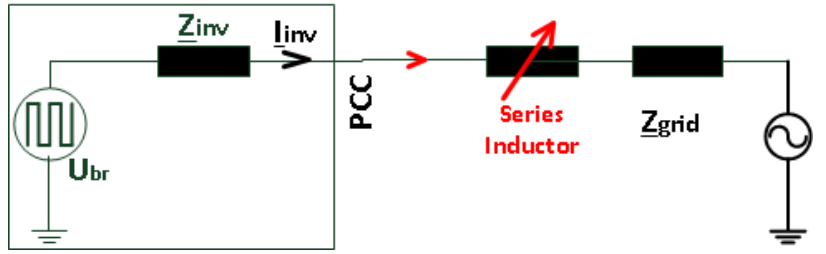

Fig. 1. Test setup for analyzing the effect of grid impedance variations on the current spectrum with invert

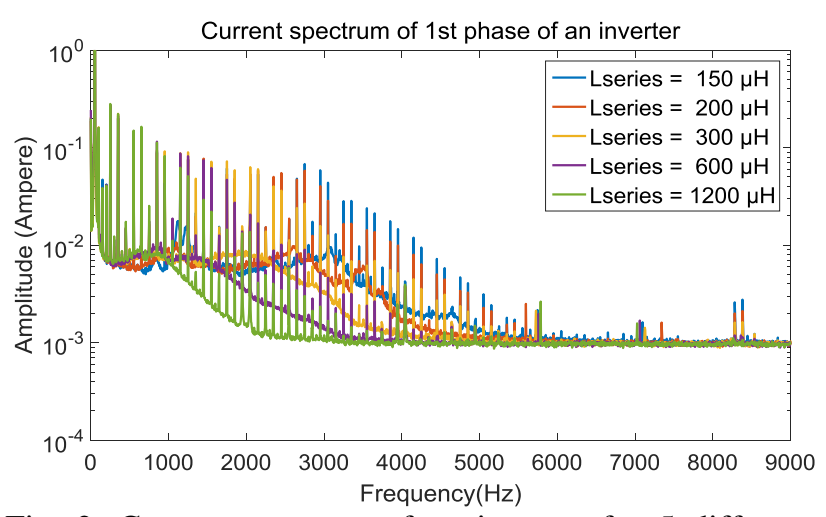

Fig. 2. Current spectrum of an inverter for 5 different values of series inductance

Fig. 2 shows a significant change for different impedance values. With increasing series impedance values, The harmonic current spectrum and its emission peaks are shifted towards lower frequencies.

\section{B. Main Concept}

As demonstrated in [11], increasing the number of identical inverters at the grid connection point can be seen as equivalent to increasing the grid impedance for a single inverter. In other words, by operating several inverters in parallel, the voltage drop across the grid series impedance increases. The same effect would be obtained with a single inverter and larger grid impedance. As a result, when $\mathrm{N}$ identical inverters are connected in an identical manner to a PCC, the dynamic behavior of the system can be analyses by modelling solely one inverter connected to a grid with an impedance $\mathrm{N}$ times higher than the real impedance of the grid. This is illustrated in Fig. 3 and will be referred to as the equivalent grid impedance in this paper.

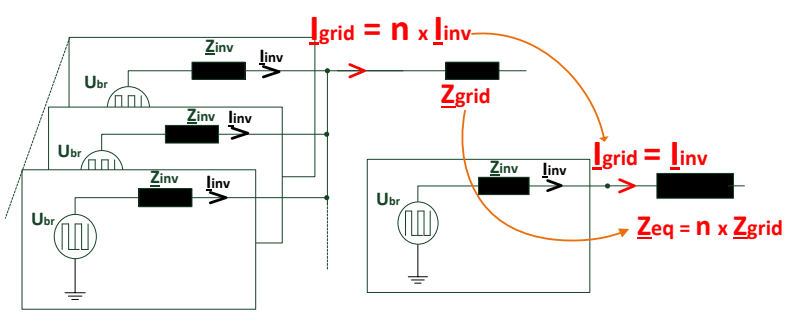

Fig. 3. $\mathrm{N}$ identical parallel operated inverters and the corresponding single inverter model with equivalent grid impedance

As discussed in section 2, the IEC 61400-21 defines the current measurement of a single inverter to predict the harmonic content of total current at the PCC of identical parallel connected inverters. The goal of this paper is to find the right basis for measurement of the single inverter with the proper equivalent grid impedance so that the predicted values by the summation law are as close as possible to the measured values at the PCC.

The discussed considerations will be applied in test bench measurements. If the theory is true, the current spectrum of four inverters connected in parallel to the grid with a series impedance $\mathrm{Z}$ should be comparable to the spectrum of a single inverter connected to the grid with a series 
inductance of $4 \times \mathrm{Z}$ after normalization of the amplitude, as shown in Fig. 4.

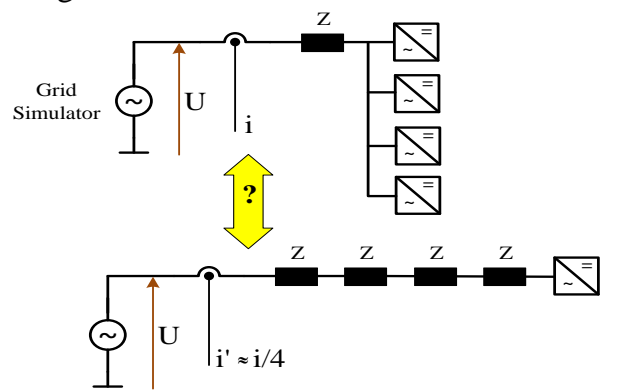

Fig. 4. Equivalent Impedance concept applied to harmonic summation

\section{Validation via Lab Measurements}

\section{A. $\quad$ Test setup}

Several analyses are carried out to examine the assumption of the equivalent grid impedance. Single line diagram of the installed test bench is illustrated in Fig. 5.

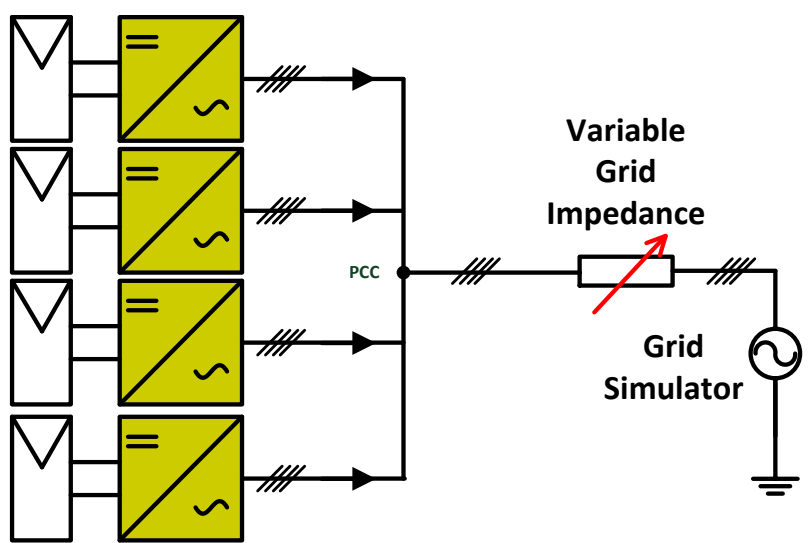

Fig. 5. Installed test bench with variable grid impedance.

The inverters for this test bench are four identical $50 \mathrm{~kW}$, three-phase, four-wire, transformer-less inverters. The grid simulator operates as a pure sinusoidal voltage source thus avoiding effects due to background distortion. In order to avoid impedance unbalance between the phases, length of the cables for the three phases and likewise for the neutral are the same. The same variable grid inductance is applied also for the neutral wire, as shown in Fig. 6.

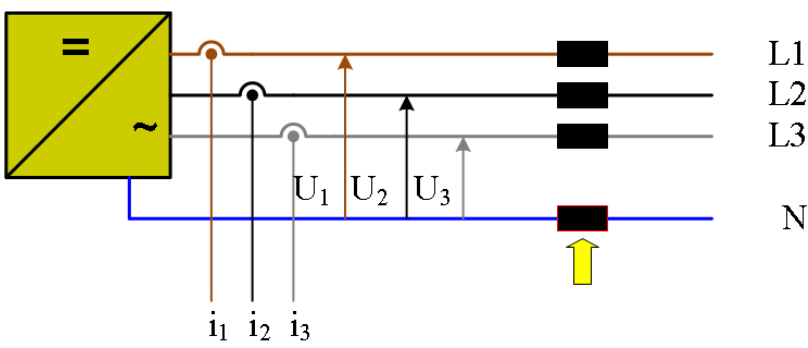

Fig. 6. Four-wire, grid connection with variable grid impedance also on neutral wire.

Inverters are supplied by four identical DC-Sources programmed with an I-V curve according to [16].

The measurement sample rate is set to $50 \mathrm{kHz}$. Currents on each phase are measured synchronously for each inverter using Rogowsky coils. At the PCC and for the first inverter, currents are additionally measured with the high precision Hal-effect current transducers. Phase-to-neutral voltages are measured at the PCC and at the grid simulator terminals.

Since large-scale PV power plants are usually connected to medium or high voltage grids, which show mainly inductive character, inductors where chosen as grid series impedances. There are four $600 \mu \mathrm{H}$ inductors available for each phase and also for the neutral, which can realize seven different values for grid equivalent impedance between $150 \mu \mathrm{H}$ and $2400 \mu \mathrm{H}$.

According to the number of parallel operated inverters and various grid impedance values 28 scenarios are possible. For each measurement, the corresponding short circuit ratios $\left(\mathrm{S}_{\mathrm{sc}} / \mathrm{S}_{\mathrm{n}}\right)$ are calculated and used to introduce it in Table I. Short circuit ratio (SCR) variates by two factors of number of parallel inverters and applied series impedance. It is obvious that if the impedance value is higher or more inverters are operating in parallel, a lower SCR is obtained and vice versa.

Table I. Achievable scenarios with the installed test bench and the resulting short-circuit ratio SCR

\begin{tabular}{|c|c|c|c|c|}
\hline $\begin{array}{c}\text { Inverter No. } \\
\text { in parallel }\end{array}$ & 1 & 2 & 3 & 4 \\
\hline 150 & & & & \\
\hline 200 & 50,52 & 25,26 & 16,84 & 12,63 \\
\hline 300 & 33,68 & 16,84 & 11,23 & 8,42 \\
\hline 600 & 16,84 & 8,42 & 5,61 & 4,21 \\
\hline 1200 & 8,42 & 4,21 & 2,81 & 2,10 \\
\hline 1800 & 5,61 & 2,81 & 1,87 & 1,40 \\
\hline 2400 & 4,21 & 2,10 & 1,40 & 1,05 \\
\hline
\end{tabular}

\section{B. Transfer function of the whole system calculated at $P C C$}

The AC voltage simulator which is used in this test bench is capable of adding a harmonic component with a favorite frequency to the main $50 \mathrm{~Hz}$ sinusoidal voltage, as shown in Fig 7. This provides the possibility to perform a so called impedance spectroscopy. For that a small perturbation voltage is added to the fundamental frequency of the voltage, provided by the $\mathrm{AC}$ grid simulator. The perturbation voltage is swept over a certain frequency range. The current response to the specific frequency of the perturbation voltage is measured at the same point and then the ratio for $\mathrm{I} / \mathrm{U}$ is calculated for each frequency component. This procedure can eventually deliver the frequency dependent transfer function at the measurement point. It is important to mention that the U/I do not represent the impedance of the inverter, while the inverter itself has internal sources of harmonic which might add up to or subtract from the emulated frequency component via the grid simulator. 


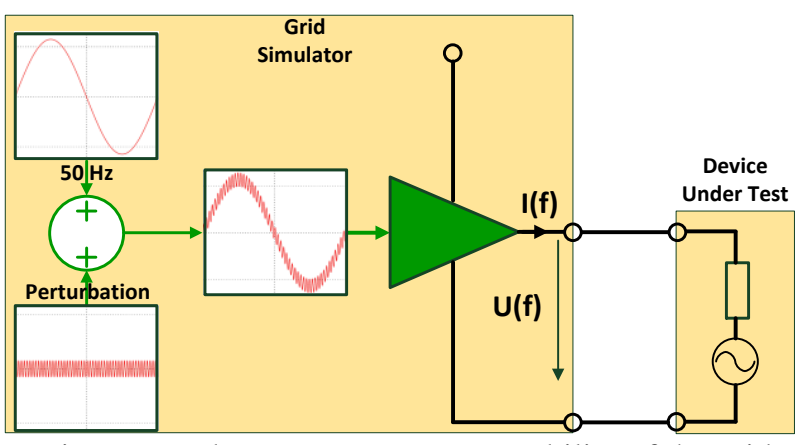

Fig 7. Impedance spectroscopy capability of the grid simulator in test bench

Performing the impedance spectroscopy and measuring at PCC, the transfer function of the whole test bench including cables and series impedances can be observed.

To have an apparent illustration of the system transfer function it is better to filter the frequency range for positive sequence frequencies. Transfer function is calculated for the scenarios listed in Table II.

Table II. Scenarios for comparison of transfer function of the whole Test bench

\begin{tabular}{|c|c|c|c|}
\hline $\begin{array}{c}\text { Scenario } \\
\text { Nr. }\end{array}$ & SCR & $\begin{array}{c}\text { Series Inductor } \\
(\mu \mathrm{H})\end{array}$ & $\begin{array}{c}\text { Inverts } \\
\text { No. }\end{array}$ \\
\hline 1 & 16.84 & 600 & 1 \\
\hline 2 & 16.84 & 300 & 2 \\
\hline 3 & 16.84 & 200 & 3 \\
\hline 4 & 16.84 & 150 & 4 \\
\hline 5 & 22.45 & 150 & 3 \\
\hline 6 & 33.68 & 150 & 2 \\
\hline 7 & 67.35 & 150 & 1 \\
\hline
\end{tabular}

Fig. 8 shows the U/I ratio of the whole test bench for the first 4 scenarios in Table II. As expected according to section 3 , the four scenarios with the $\mathrm{SCR}=16.84$ follow almost the same pattern in gain and phase diagrams. The three other measurements have been performed with the same series inductor $(150 \mu \mathrm{H})$ but with decreasing number of operated inverters, which leads to higher SCR values. This results in higher values for the I/U-ratio and shifts its peak towards higher frequencies as it can be seen in Fig. 9.
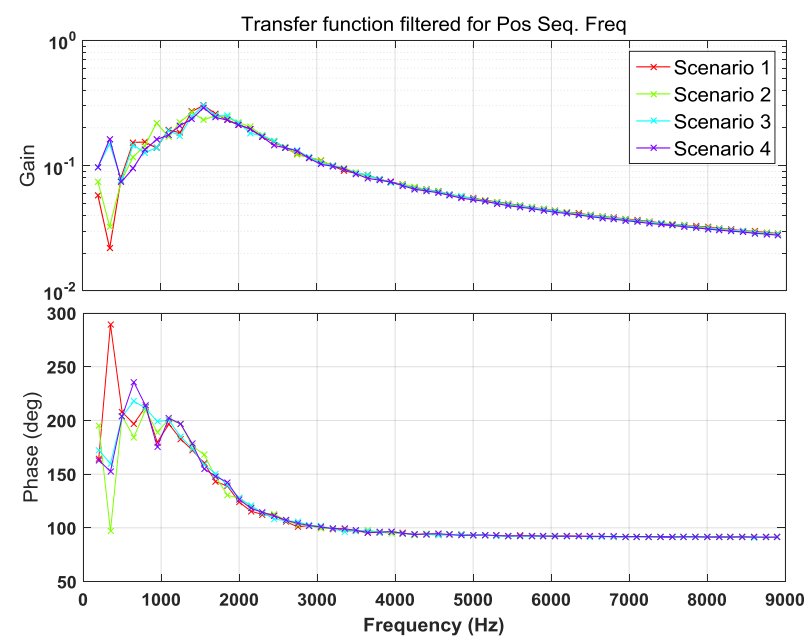

Fig. 8. I/U ratio measured at PCC for 1 to 4 inverters in Parallel and constant SCR $=16.84$

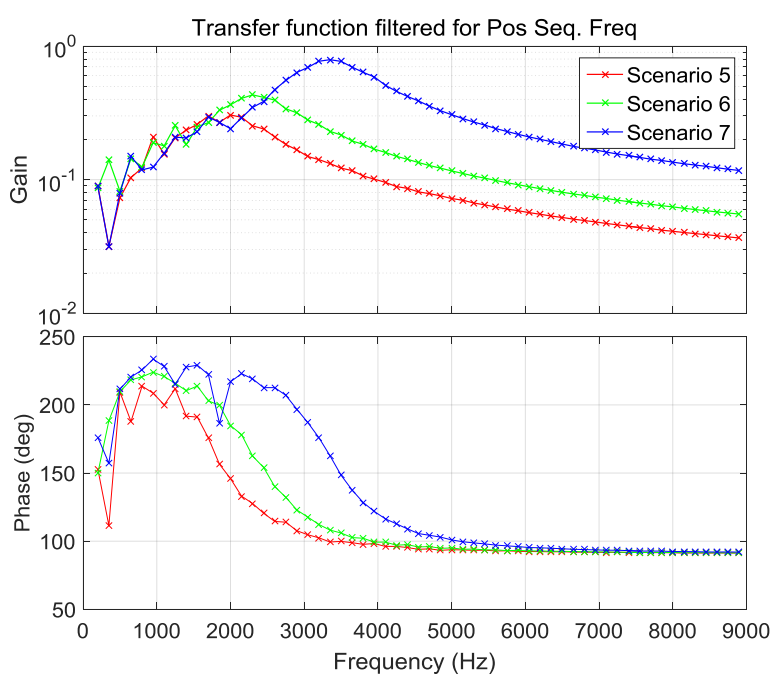

Fig. 9. I/U ratio measured at PCC with 1 to 3 inverters and a constant Series Inductance of $150 \mu \mathrm{H}$

\section{Spectrum of one single inverter}

Observing the current spectrum, it can be noticed that the peaks occur mostly at $6 n \pm 1$ harmonic components and the overall behavior of the spectrum is followed also by these components, as seen in Fig. 10. For a clearer visualization of current spectrum, just these components are shown for the rest of illustrations.

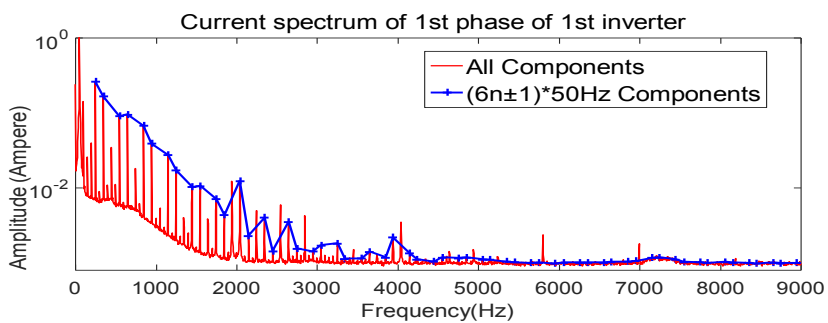

Fig. 10. Unfiltered Current spectrum vs current spectrum filtered for $6 \mathrm{n} \pm 1$ harmonics

Current spectrum of the measurements with same and different SCR and a no load measurement are shown in Fig. 11. No load measurement proves that the components with amplitude smaller than about $1.15 \mathrm{~mA}$ are under the noise limit of measurement devices, as a result they shall not be considered for further analysis.

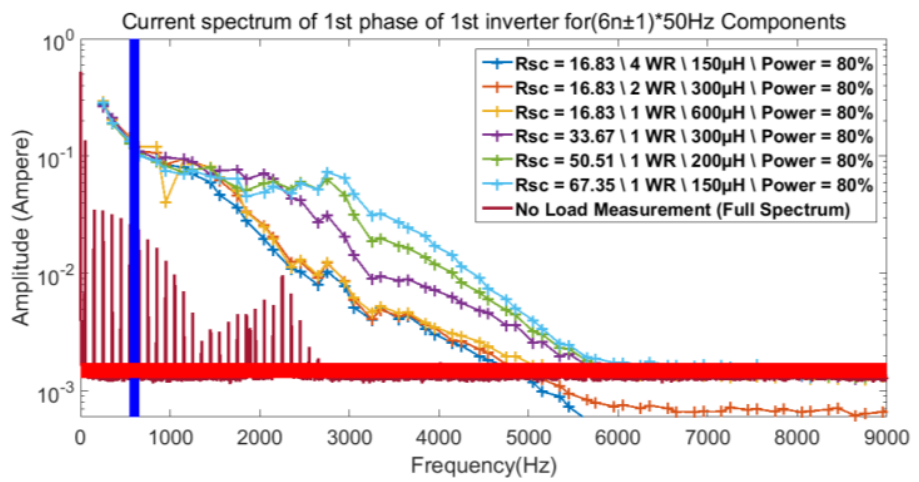

Fig. 11. Current spectrum for measurements with different SCR and no-load measurement 
Furthermore, it is visible that, for the lower frequency harmonic components up to $550 \mathrm{~Hz}$ the amplitude of current spectrum is independent of the grid equivalent impedance, which means that the inverter acts as a current source in this frequency range. For the other components to the right of blue line and at the top of the red line, the behavior of the inverter can be considered as an impedance combined with an internal voltage source as proposed in [7] leading to an harmonic current emission that is dependent of the applied grid equivalent impedance.

On the other hand it is known that the current spectrum of a single inverter is utilized to predict the spectrum of identical parallel inverters. Therefore, investigation of the current spectrum of one inverter when it is in single operation and when operated in parallel with more inverters will help to better understand how the measurements with adjusted grid equivalent impedance can improve the results of the summation law. Scenarios for this comparison that satisfy the criterion illustrated in Fig. 4 are presented in Table III.

Table III. Scenarios for comparison of spectrums

\begin{tabular}{|c|c|c|c|c|}
\hline $\begin{array}{c}\text { Scn. } \\
\text { Nr. }\end{array}$ & SCR & $\begin{array}{c}\text { Series } \\
\text { Ind. } \\
(\mu \mathrm{H})\end{array}$ & $\begin{array}{c}\text { Inverters } \\
\text { No. }\end{array}$ & Scenario description \\
\hline 1 & 4.21 & 1200 & 2 & PCC meas. as Reference \\
\hline 2 & 4.21 & 2400 & 1 & Adjusted grid Eq. Imp. \\
\hline 3 & 8.42 & 1200 & 1 & Unadjusted grid Eq. Imp. \\
\hline 4 & 16.84 & 150 & 4 & PCC meas. as Reference \\
\hline 5 & 16.84 & 600 & 1 & Adjusted grid Eq. Imp. \\
\hline 6 & 67.35 & 150 & 1 & Unadjusted grid Eq. Imp. \\
\hline
\end{tabular}

Each set of scenarios identified with a red box in Table III includes three scenarios:

- The first scenario is a measurement at PCC while operating two or more inverters in parallel with a defined series inductance.

- The second scenario is a measurement performed with only one inverter but keeping the SCR identical to the first scenario by adapting the series inductance.

- The third scenario is a measurement performed with only one inverter and the same series inductance as in the first scenario, leading to a different SCR.

The PCC is selected as current measurement point for these illustrations and the current is always normalized according to the number of parallel operated inverters. Fig. 12 visualizes the comparison of these three scenarios for each set (red box) and shows how the conformity of spectrums is improved by adjusting the grid equivalent impedance, i.e. the SCR.

The diagrams in Fig. 12 and shown for the frequencies up to $6 \mathrm{kHz}$ because higher frequency components will be lower than the noise limit and it is visible that the measurements with higher equivalent impedance enter the noise limit in higher frequencies.

As expected, the adjustment of the grid equivalent impedance leads to a better estimation of the current emission that can be expected when scaling up the emission of a single inverter to several inverters in parallel.
Current spectrum of 1 st phase of 1 st inverter for $(6 n \pm 1)^{\star} 50 \mathrm{~Hz}$ Components

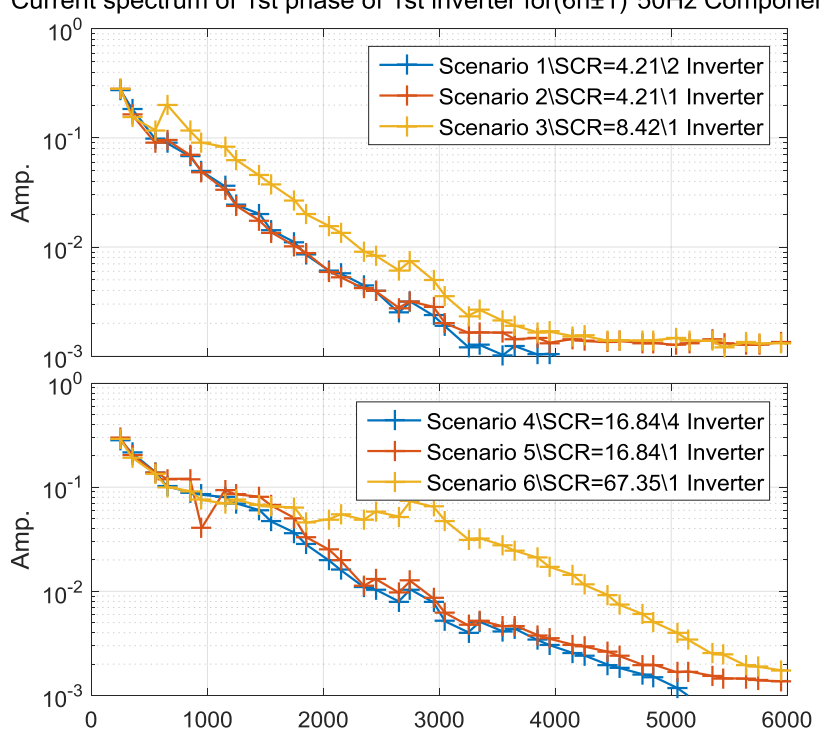

Fig. 12. Comparison of current spectrum of one inverter for different set of scenarios in Tabel III.

\section{Summation law}

It is now expected that the grid equivalent impedance can also improve the results of the summation law. In order to implement the summation law following the implications of IEC 6100-4-7 [12], the harmonics and inter-harmonics are grouped via subgrouping procedure and then aggregated over a period of one minute which corresponds to 300 DFT windows.

For application of summation law, the exponents recommended by IEC 61400-21[1] are used.Fig. 13 shows results of summation law for harmonics and interharmonics for the first set of scenarios in Table III. In this depiction, harmonic emission at the PCC of parallel inverters in scenario one is compared to the results of summation law applied to the PCC measurements for the second and third scenarios for $\mathrm{N}=2$ in Equation 1. Thus the effect of adjusting the equivalent grid impedance can be seen in Fig. 13. The higher order harmonic components are not illustrated because these components are already below the noise limit. According to the observations, results from Summation law and PCC measurements remain fairly the same up to the frequency of about $550 \mathrm{~Hz}$, which is because of the current source behavior of the inverter. Beyond this limit, it is possible to observe the optimization effect of grid equivalent impedance. Adjustment of the grid equivalent impedance improves the results of summation law for harmonics and inter-harmonics, although there are still discrepancies when comparing to the measurement at PCC. 

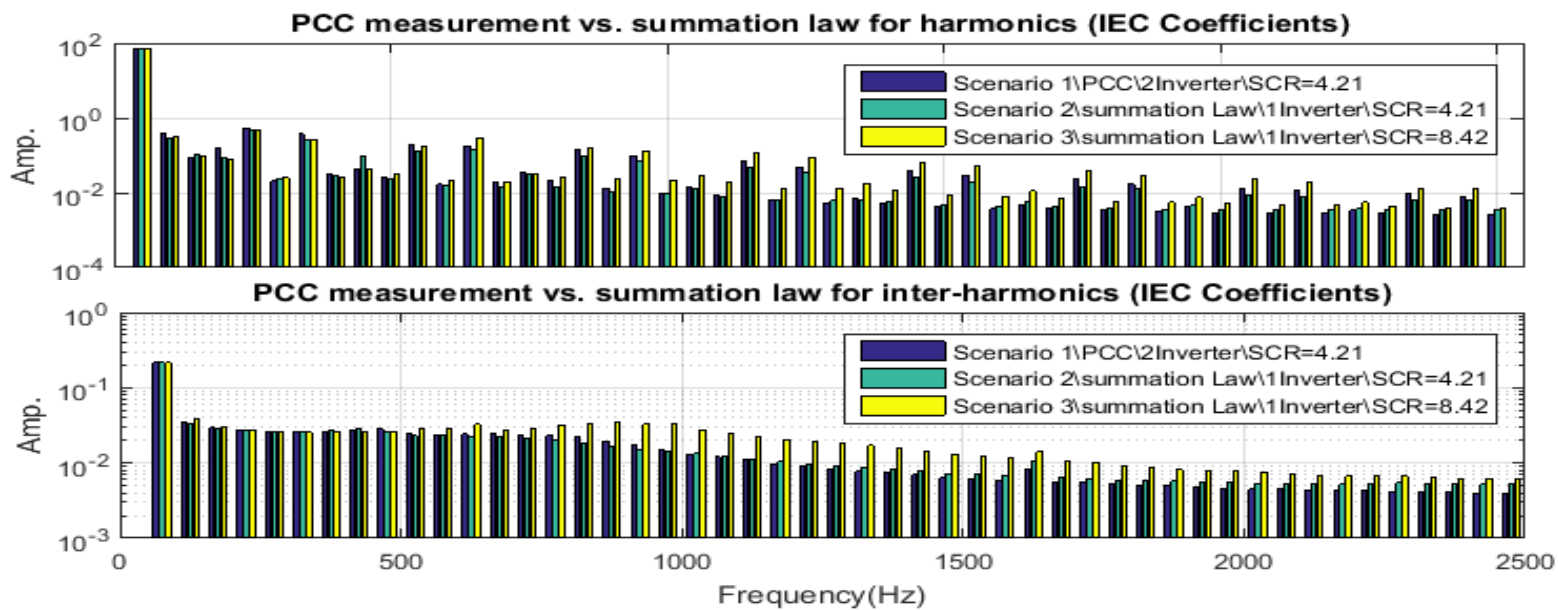

Fig. 13. Comparison of the summation law for adjusted and unadjusted grid equivalent impedance

Since the spectrum illustrations show a better conformity than the summation results, these discrepancies might origin from the applied coefficients for summation law. Thus, it could be possible to achieve better conformity by tuning the coefficients which are recommended by IEC standard or other grid codes. Assessing summation coefficients is out of framework of this paper but it is recommended for further investigations.

\section{Conclusion}

This paper proposes using the grid equivalent impedance to improve the performance of summation law for harmonics. A test setup was installed and the proposed theory was investigated via evaluation of the transfer function of the system and comparisons of frequency spectrum. The observations proved firmly that application of grid equivalent impedance is essential to predict the overall harmonic emission of multiple parallel operated inverters at their PCC. This could eventually be used for a better prediction of emission at PCC of PV parks regarding emissions and the response to background distortion.

In order to achieve an even better result using summation law an optimization of the used summation exponents shall be investigated.

\section{Acknowledgement}

Parts of this work were financed by the German federal ministry for economic affairs and energy under the project "Netzharmonie" (0325757F).

\section{References}

[1] IEC 61400-21, Measurement and assessment of power quality characteristics of grid connected wind turbines, Edition 2, 2006.

[2] Jan Meyer et. al., "Survey on International Practice of Calculating Harmonic Current Emission Limits", ICHQP 2016

[3] IEC 61000-3-6, Limits - Assessment of emission Iimits for the connection of distorting installations to MV, HV and EHV power systems, Edition 2.0 2008-02

[4] A.E. Emanuel and S.R. Kaprielian, "Contribution to the Theory of Stochastically Periodic Harmonics in Power Systems,"
IEEE Transactions on Power Delivery, vol.1, no.3, pp.285-293, Jul. 1986.

[5] W.G. Sherman, "Summation of Harmonics with Random Phase Angles," Proceedings of the Institution of Electrical Engineers, vol.119, no.11, pp.1643-1648, Nov. 1972.

[6] Y. Baghzouz and O.T. Tan, "Probabilistic Modelling of Power System Harmonics," IEEE Transactions on Industry Applications, vol.IA-23, no.1, pp.173-180, Jan. 1987.

[7] K. Malekian, A. Gohlich, L. Pop, and W. Schufft, "A probabilistic approach to analyze and model the simultaneity of power produced by wind turbines in a wind farm" 6th International Conference on Information Technology and Electrical Engineering (ICITEE), pp.1-7, Oct. 2014.

[8] K. Reusel, S. Bronckers "Summation rule for wind turbines' harmonics challenged by measurements", ICHQP 2016

[9] F. Medeiros, D.C. Brasil, P.F. Ribeiro, C. A. G. Marques and C. A. Duque, "A New Approach for Harmonic Summation Using the Methodology of IEC 61400-21", ICHQP 2010

[10] F. Ackermann, N. Bihler, S. Rogalla, "Stability Prediction and Stability Enhancement for Large-Scale PV Power Plants", PEDG 2016

[11] J. Agorreta, M. Borrega, J. López and L. Marroyo, "Modeling and control of N-paralleled grid-connected inverters with LCL filter coupled due to grid impedance in PV plants," in Power Electronics, IEEE Transactions on (Volume:26 , Issue: 3 ), 2011.

[12] IEC 61000-4-7 Electromagnetic compatibility (EMC) Part 4-7: Testing and measurement techniques - General guide on harmonics and inter-harmonics measurements and instrumentation, for power supply systems and equipment connected thereto, $2002+\mathrm{A} 1: 2008$

[13] F. Ackermann, G. Dötter, R. Singer, S. Rogalla, title in German " Messtechnische Überprüfung der Summationsgesetze für Oberschwingungsemissionen", ZNE 2014

[14] F. Santjer, B. Weise, T. Pausch, J. Brombach, "Aspects for improvement of measurement and assessment procedures of harmonic emission of wind power plants", DEWEK 201

[15] S. Rogalla, u. a., „Schwierige Bewertung von Oberschwingungsemissionen - Herausforderungen bei der OSVermessung von großen Erzeugern am realen Netz",Zukünftige Stromnetze für Erneuerbare Energien, Berlin, 2014

[16] Deutsche Kommission Elektrotechnik Elektronik Informationstechnik, DIN EN 50530 "Overall efficiency of grid connected photovoltaic inverters", Berlin: Beuth Verlag, 2013. 